\title{
Efecto inmediato de la auto-liberación miofascial en la superficie plantar sobre la musculatura isquiosural en futbolistas
}

\section{Immediate effect of myofascial self-release on the plantar surface over the hamstring musculature in football players}

\author{
Juan Manuel Barradas Romero, Samuel Gómez Torres, Aurelio Montero Duarte, \\ Rafael Bogas Arrebola y Ramón Chacón Cuberos
}

Facultad de Educación, Psicología y Ciencias del Deporte, Universidad de Huelva (España)

\begin{abstract}
Resumen: Las adherencias en ciertas zonas musculares generan tensiones en otras musculaturas de la misma cadena muscular. El objetivo del estudio es probar el efecto inmediato de la "Auto-liberación Miofascial" (SMR) con la técnica de pelota de golf en la fascia plantar en deportistas federados de "Fútbol 11" (FU) y "Fútbol Sala" (FS) y su incidencia sobre la musculatura isquiosural. Además, se analizó las diferencias entre cada modalidad. El diseño es de tipo experimental longitudinal, con una muestra de 20 deportistas federados. Los resultados muestran una mejora en la longitud alcanzada en el "Test Sit and Reach" (SRT) de forma significativa la SMR entre "Grupo Control" (GC) y "Grupo Experimental" (GE). Entre modalidades no se aprecian diferencias significativas. Se concluye que existe un efecto inmediato de la SMR con la técnica de pelota de golf en la fascia plantar sobre la musculatura isquiosural. No existiendo diferencias significativas entre modalidades.
\end{abstract}

Palabras Claves: Auto-liberación miofascial; Rango de movimiento; Isquiosurales; Fascia plantar.
Abstract: Adhesions in certain muscle areas generate tension in other muscles of the same muscle chain. The aim of the study is to test the immediate effect of the "Self-myofascial release" (SMR) with the golf ball technique on the plantar fascia in federated sportsmen of "Football 11" (FU) and "Futsal" (FS) and its influence on hamstring musculature. Besides, the differences between each modality have been analysed. The design is of the longitudinal experimental type, with a sample of 20 federated sportsmen. The results show significant improvement in the length reached in the "Sit and Reach Test" (SRT) after the SMR between "Control Group" (GC) and "Experimental Group"(GE). There are no significant differences between modalities. It is therefore concluded that there is an immediate effect of SMR with the golf ball technique on the plantar fascia over the hamstring muscle. There are no significant differences between modalities.

Key Words: Self myofascial release; Range of motion; Hamstrings; Plantar fascia.

\section{Introducción}

La flexibilidad es definida por Sainz, Cejudo, Ayala y Santonja (2015) como "la capacidad intrínseca de los tejidos corporales que determinan el máximo rango de movimiento sin llegar a la lesión deportiva". Mediante test es posible obtener una valoración cuantitativa de la flexibilidad, y aquí es donde aparece el término "Amplitud De Movimiento" (ADM) o "Range of Motion" (ROM) (Merino, López, Torres y Fernández, 2011b). Estos conceptos representan una medición indirecta de la flexibilidad muscular (Sainz et al., 2015).

En la actualidad, se está teniendo en cuenta la flexibilidad como un componente de gran importancia en el rendimiento deportivo (Sainz et al., 2015). Por ello, se deben incluir métodos para obtener una idónea flexibilidad muscular en los atletas, con el fin de que no presenten una hipomovilidad en las acciones deportivas, siendo esta un factor que pueda derivar en una lesión (Raya-González y Estévez-Rodríguez, 2016).

Dirección para correspondencia [Correspondence address]: Juan Manuel Barradas Romero. Facultad de Educación, Psicología y Ciencias del Deporte, Universidad de Huelva (España).

E-mail: juanmanuel.barradas@alu.uhu.es
La "Auto-Liberación Miofascial" (SMR) es un método de ganancia aguda de ROM. Ferreira (2015), indica que este método se basa en la conciencia del cuerpo como una unidad funcional, en la que los músculos se interconexionan entre sí formando cadenas musculares. El tejido que realiza dichas interconexiones, de la que depende el equilibrio global y el funcionamiento general del individuo, son las fascias, y posee las funciones de conectar, suspender, proteger, rodea, soporta y divide (Ferreira, 2015). Ya sea por un periodo de inactividad o debido a una lesión, los sujetos que no favorecen la continua elongación-contracción de las fascias pueden verse sometidos una pérdida de elastina, haciéndolas menos flexibles y a adherirse unas a otras (Campignion, 2001).

La terapia de SMR se fundamenta en los principios que desarrolló Barnes en 1997 sobre la liberación miofascial, pero esta no requiere de la intervención del personal especializado, sino que el individuo mediante la utilización de diversos implementos como pelotas, rodillos o el "foam roller" la puede realizar. Este método pretende liberar el tejido fascial adherido o con restricciones, ejerciendo presión sobre la superficie corporal (Ferreira, 2015) 
Diversos estudios científicos constatan la eficacia de la SMR para la ganancia de ROM sobre la zona muscular manipulada (DeBruyne, Dewhurst, Fischer, Wojtanowski, y Durall, 2017; Junker y Stöggl, 2015), del mismo modo que para otras zonas musculares remotas de la misma cadena miofascial (Do, Kim, y Yim, 2018; Grieve et al., 2015; Merino, Mayorga, Fernández y García, 2011a). Dicha técnica parece no interferir en el rendimiento deportivo del atleta (Beardsley y Skarabot, 2015; Kalichman y Ben-David, 2017), ayudan a mejorar de la función arterial (Okamoto, Masuhara y Ikuta, 2014) y a la recuperación mediante la atenuación del dolor tras el ejercicio (Macdonald, Button, Drinkwater y Behm, 2014).

La SMR puede ser un buen método para reducir el acortamiento que tiende a sufrir la musculatura isquiosural, esta carencia se debe a sus propiedades, la función de control postural y a las continuas fuerzas de tensión a las que están sometidos (Medeiros, Cini, Sbruzzi y Lima, 2016).

La musculatura isquiosural es un tanto problemática debido al gran número de lesiones que se producen (Ekstrand, Waldén y Hägglund, 2011). Ekstrand, Waldén y Hägglund (2016) observaron a 36 clubes de "Fútbol" (FU) de 12 países diferentes durante 13 ańos. Los resultados indicaron que el $22 \%$ de los jugadores padecieron al menos una lesión en la musculatura isquiosural durante una temporada, y la tendencia muestra un aumento lesivo del 2,3\% cada ańo, siendo más acentuada en los entrenamientos, con un aumento del $4 \%$. Estos resultados pueden tener su relación con las exigencias en el juego actual. Barnes, Archer, Hogg, Bush y Bradley, (2014) y Bush, Barnes, Archer, Hogg y Bradley, (2015) concluyen en sus estudios que la distancia de carrera en un partido de FU se ha incrementado con el paso de los ańos, además, se observa la misma tendencia en número y distancia de los sprints.

En cuanto al "Fútbol Sala" (FS), Martínez-Riaza, Herrero-González, López-Alcorocho, Guillen-García y FernándezJaén (2017), realizaron un seguimiento al equipo nacional español de FS durante 5 temporadas. Los resultados muestran que la zona más lesiva fue los isquiosurales.

Algunos autores observan que no existe una correlación entre la flexibilidad de los músculos isquiosurales y las lesiones musculares en esa región (Van Doormaal, Van der Horst, Backx, Smits y Huisstede, 2017), aunque otros autores si lo indican en mayor o menor medida como un factor de riesgo lesivo (Witvrouw, Danneel, Asselman, D’Tienes y Cambier, 2003; Van Dyk, Farooq, Bahr y Witvrouw, 2018). También existen estudios ponen el foco en otras patologías derivadas de la carencia de flexibilidad en músculos de los isquiosurales, este déficit puede generar limitaciones en el ROM de las articulaciones de la rodilla y la cadera (Mason et al., 2016), pudiendo dar lugar a una biomecánica alterada y a su vez disfunciones articulares (Joshi, Balthillaya y Prabhu, 2018), también puede reducir la función de proporcionar estabilidad a dichas articulaciones (Moon, Jung, Sik y Cho, 2017), además de generar desequilibrios musculares importantes produciendo lesiones musculares y dolor lumbar (Medeiros et al., 2016).

En base a lo expuesto, y debido a la poca existencia de bibliografía referente a esta técnica de SMR sobre deportistas federados, el objetivo de esta investigación tratará de probar el efecto inmediato de la SMR con la técnica de pelota de golf en la fascia plantar en deportistas federados de FS y FU, y su incidencia sobre la musculatura isquiosural. Además, desea analizar las diferencias entre cada modalidad deportiva (FU y FS) después de ser sometidos a la SMR.

\section{Material y método}

\section{Diseño y participantes}

Para desarrollar el estudio, se empleó un diseño longitudinal cuasiexperimental con grupo control equivalente. Participaron un total de 20 hombres deportistas federados de nivel provincial, que practicaban dos modalidades diferentes FU y FS elegidas por conveniencia. Por un muestreo aleatorio simple, 5 sujetos de cada modalidad fueron distribuidos en el "Grupo Control" (GC) y otros 5 de cada modalidad en el "Grupo experimental" (GE).

Para formar parte de la muestra se establecieron unos requisitos de inclusión-exclusión. Algunos de estos requisitos han sido utilizados por otros autores como Merino et al. (2011a) para desarrollar un estudio similar. Los sujetos aptos debieron tener entre 18 y 30 años $(\bar{X}=22.80$; DT $=$ 2.726), no padecer ninguna patología que se pudiera agravar durante la realización del estudio, no presentar limitaciones musculoesqueléticas, no presentar agujetas en el momento de la obtención de los datos y no presentar dolor raquídeo o coxofemoral que pudiera limitar la ejecución del "Test Sit and Reach” (SRT).

Todos los sujetos que consintieron participar en el estudio debieron entregar un documento de consentimiento informado debidamente firmado.

\section{Variables}

Las variables empleadas en el presente estudio han sido las siguientes:

- Edad. Establece la edad de cada sujeto que ha participado en el estudio.

- Modalidad deportiva. Determina el tipo de práctica deportiva que ejecuta cada participante. Se codifica en $0=$ FS y $1=$ FU 


\section{Instrumentos}

Para llevar a cabo el estudio se necesitó ciertos instrumentos.

- Cuestionario AD HOC: herramienta de elaboración propia para determinar la modalidad deportiva y los requisitos de inclusión-exclusión de la muestra del estudio.

- Cajón SRT: instrumento para realizar el SRT. Este test fue desarrollado por Well y Dillon y debido a su fácil procedimiento ha sido incluido como una de las pruebas más extendidas en la medición de flexibilidad de la musculatura lumbar e isquiosural. Presenta una validez moderada-alta para determinar la flexibilidad de la musculatura isquiosural $(\mathrm{r}=0.37-0.77)$ para hombres y $(r=0.37-0.85)$ mujeres (Ayala, Sainz, Croix y Santonja, 2012).

- Pelotas de golf Inesis Distance 100: implemento para ejecutar la SMR.

- Banquetas: asiento para facilitar la realización de la SMR.

\section{Procedimiento}

Para su realización, se comenzó solicitando la participación a ciertas entidades deportivas y a sus correspondientes deportistas. Seguidamente, se procedió a la obtención de los consentimientos informados y a cumplimentar los cuestionarios Ad-Hoc para la inclusión-exclusión de este.

Partiendo del estudio llevado a cabo por Merino et al. (2011a), los sujetos individualmente ejecutaron en un inicio dos mediciones del SRT, entre cada medición se deja un tiempo de un minuto de descanso.

Para realizar correctamente la medición se explicó al sujeto la posición de partida, es decir, sentado, con las rodillas extendidas, los pies $90^{\circ}$ de flexión y con la superficie plantar en contacto permanente con el cajón de SRT. A partir de esta posición, se le indica al sujeto que puede proceder de forma lenta y progresivamente a la flexión del tronco, manteniendo en todo momento las piernas y los brazos extendidos. Una vez llegue al máximo de dicha flexión debe mantenerse 2 segundos.

Seguidamente los sujetos que pertenecían al grupo GE se sentaron en una banqueta y colocando una pelota de golf debajo de cada pie, debieron hacerlas rodar por toda la superficie plantar con una intensidad/presión media durante cinco minutos. En cambio, los sujetos del GC no realizaron ningún tipo de actividad, simplemente permanecieron sentados en la misma posición que los sujetos del GE al realizar la SMR, con ambos pies sobre el piso. Transcurrido el tiempo, nuevamente realizaron otras dos mediciones del SRT con el mismo protocolo que al inicio del estudio.

Se requirió que los sujetos asistieran en ropa deportiva (pantalón corto) y descalzos en todo momento.
La supervisión del estudio fue realizada por investigadores en pleno conocimiento del procedimiento. En todo momento se ha respetado la confidencialidad de los participantes, respetado así la declaración de Helsinki para normas éticas de investigación y se aseguró a todos los participantes que los datos serían tratados con fines científicos y académicos.

\section{Análisis de datos}

Se emplearon descriptivos básicos para obtener la media " $\bar{X}$ " y la desviación típica " $D T$ ". Para las correlaciones en cada grupo se empleó $\mathrm{T}$ de student para muestras relacionadas, además, se incluyó la d de Cohen. También, se llevó a cabo $\mathrm{T}$ de student para muestras independientes con el objetivo de observar las correlaciones en cada una de las modalidades. En análisis estadístico se realizó mediante el software IBM $^{\circledR}$ SPSS Statistics 20.

\section{Resultados}

En los análisis realizados, se encontraron diferencias significativas entre los pretest y los post-test en el GE ( $p=0.000)$, de igual modo que en los pretest y los post-test del GC que no realizó la SMR ( $\mathrm{p}=0.000)$ (Tabla 1$)$.

El GC mostró un aumento de las medias a medida que realizaron los test, pasando de $(-2.80)$ a $(-1.57)$ en el primer y segundo pretest respectivamente y de $(-0.47)$ a $(-0.12)$ en el primer y segundo post-test. El GE al igual que en el GC se presentó un aumento progresivo de las medias de (-6.70) a $(-5.06)$ en el primer y segundo pretest respectivamente y de $(-0.47)$ a $(-0.12)$ en el primer y segundo post-test (Tabla 1$)$.

En las variaciones de medias se observó en el GC variaciones de (-2.33) entre primeras mediciones, mostrando un aumento de ROM superior a la variación de medias obtenida en las segundas mediciones (-1.44). Con respecto al GE se obtuvieron variaciones de medias superiores a las del GC (-4.36) y (-4.14), produciéndose un aumento de ROM más destacado en este grupo. Al igual que en el GC, se produjo un aumento de ROM superior en la variación obtenida en las primeras mediciones (Tabla 1).

En cuanto al tamaño del efecto según Cohen (1988), se observa que el GC muestra unos tamaños del efecto pequeños, tanto entre las primeras mediciones $(0.338)$ como en las comparativas entre las segundas mediciones (0.223), siendo superior este valor en las primeras mediciones. Con respecto al GE, de igual modo que en el GC el tamaño del efecto es pequeño, pero unos datos mayores que los obtenidos en el GC y sensiblemente superiores en las primeras mediciones (0.428) que en la comparativa entre las segundas mediciones (0.402) (Tabla 1). 
Tabla 1. Tabla de comparación entre GC y GE.

\begin{tabular}{|c|c|c|c|c|c|c|c|}
\hline & Media & DT & Variación media & 95\% I.C. & $\mathrm{T}$ & Sig. (bilateral) & $d$ \\
\hline Pretest 1 (GC) & -2.80 & 7.18 & \multirow{2}{*}{-2.33} & \multirow{2}{*}[-3.19;-1.46]{} & \multirow{2}{*}{-6.121} & \multirow{2}{*}{0.000} & \multirow{2}{*}{0.338} \\
\hline Post-test 1 (GC) & -0.47 & 6.59 & & & & & \\
\hline Pretest 2 (GC) & -1.56 & 6.57 & \multirow{2}{*}{-1.44} & \multirow{2}{*}[-2.01;-0.86]{} & \multirow{2}{*}{-5.661} & \multirow{2}{*}{0.000} & \multirow{2}{*}{0.223} \\
\hline Post-test 2 (GC) & -0.12 & 6.31 & & & & & \\
\hline Pretest 1 (GE) & -6.70 & 10.50 & \multirow{2}{*}{-4.36} & \multirow{2}{*}[-5.42;-3.21]{} & \multirow{2}{*}{-9.262} & \multirow{2}{*}{0.000} & \multirow{2}{*}{0.428} \\
\hline Post-test 1 (GE) & -2.34 & 9.83 & & & & & \\
\hline Pretest 2 (GE) & -5.06 & 10.78 & \multirow{2}{*}{-4.14} & \multirow{2}{*}[-5.63;-2.64]{} & \multirow{2}{*}{-6.263} & \multirow{2}{*}{0.000} & \multirow{2}{*}{0.402} \\
\hline Post-test 2 (GE) & -0.92 & 9.78 & & & & & \\
\hline
\end{tabular}

Tanto en el GC como en el GE, se observó un incremento de las medias en el ROM de los sujetos a medida que realizan los test, siendo este incremento de ROM superior en el GE. Además, tanto el grupo que realizó la SMR como el que no la llevo a cabo presentaron aumentos en el ROM en el Post-test 1, siendo más destacada en el grupo que sí realizó la SMR (Figura 1).

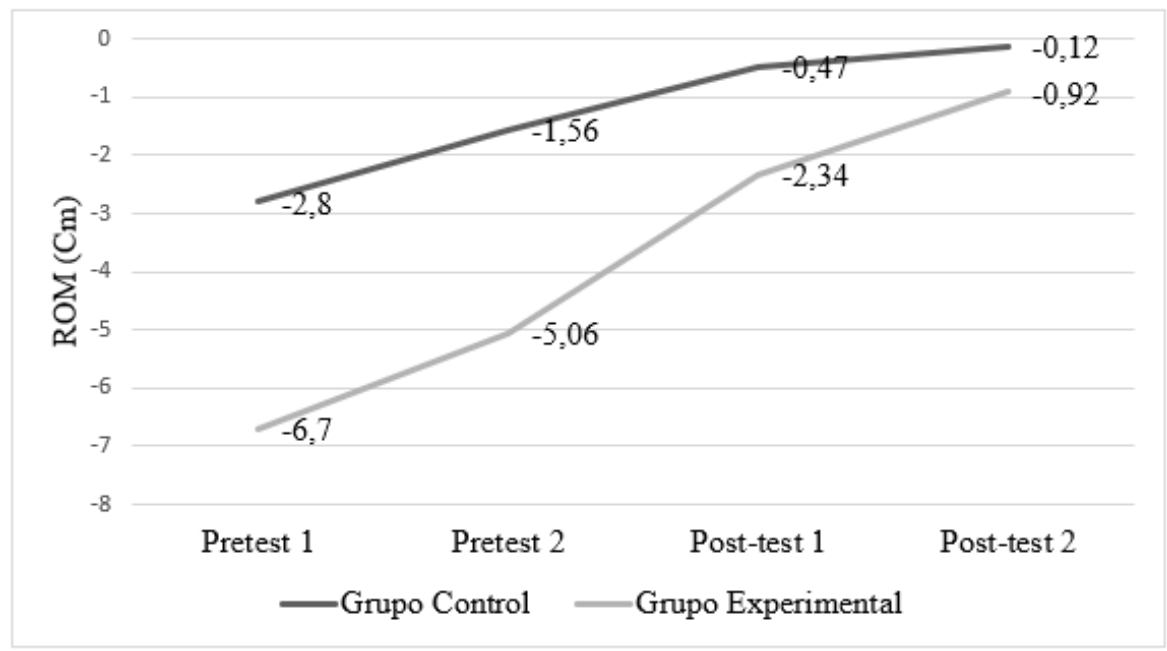

Figura 1. Comparación de medias entre GC y GE.

En cuanto a las comparaciones entre modalidad deportiva del GE, se observa diferencias significativas $(p=0.047)$ en el pretest 2, mostrando unas medias de (-11.6) y (1.48) en FU y FS respectivamente y una variación de medias de (-13.08), aumentando con respecto la variación de media del pretest 1 (Tabla 2).

Tabla 2. Tabla de comparación GE entre modalidad

\begin{tabular}{|c|c|c|c|c|c|c|c|c|}
\hline & \multirow{2}{*}{ Modalidad } & \multirow{2}{*}{ Media } & \multirow{2}{*}{ DT } & \multirow{2}{*}{$\mathrm{F}$} & \multirow{2}{*}{ Variación media } & \multicolumn{2}{|c|}{ Prueba de Levene } & \multirow{2}{*}{ Prueba T (Sig.) } \\
\hline & & & & & & $\mathrm{F}$ & Sig. & \\
\hline \multirow{2}{*}{ Pretest 1} & Fútbol Sala & -12.5 & 6.2849 & 2.8107 & \multirow{2}{*}{-11.60} & \multirow{2}{*}{4.886} & \multirow{2}{*}{.058} & \multirow{2}{*}{0.077} \\
\hline & Fútbol 11 & -0.9 & 11.1636 & 4.9925 & & & & \\
\hline \multirow{2}{*}{ Pretest 2} & Fútbol Sala & -11.6 & 5.9207 & 2.6478 & \multirow{2}{*}{-13.08} & \multirow{2}{*}{6.074} & \multirow{2}{*}{.039} & \multirow{2}{*}{0.047} \\
\hline & Fútbol 11 & 1.48 & 10.9408 & 4.8929 & & & & \\
\hline \multirow{2}{*}{ Post-test 1} & Fútbol Sala & -8.04 & 6.1366 & 2.7444 & \multirow{2}{*}{-11.40} & \multirow{2}{*}{5.743} & \multirow{2}{*}{.043} & \multirow{2}{*}{0.061} \\
\hline & Fútbol 11 & 3.36 & 9.9354 & 4.4433 & & & & \\
\hline \multirow{2}{*}{ Post-test 2} & Fútbol Sala & -6.62 & 7.0258 & 3.142 & \multirow{2}{*}{-11.40} & \multirow{2}{*}{1.835} & \multirow{2}{*}{.213} & \multirow{2}{*}{0.059} \\
\hline & Fútbol 11 & 4.78 & 9.2202 & 4.1234 & & & & \\
\hline
\end{tabular}


Aunque no presenten diferencias significativas todas las comparativas, se observó una tendencia a la ganancia de ROM tanto en la modalidad de FU como en la de FS, destacando un mayor aumento de ROM tras la SMR en el grupo que menos ROM inicial presentaba (FS). También, se mostró un mayor aumento de ROM tras la realización del pretest 2 en la modalidad deportiva que mayor ROM inicial disponían (FU) (Figura 2).

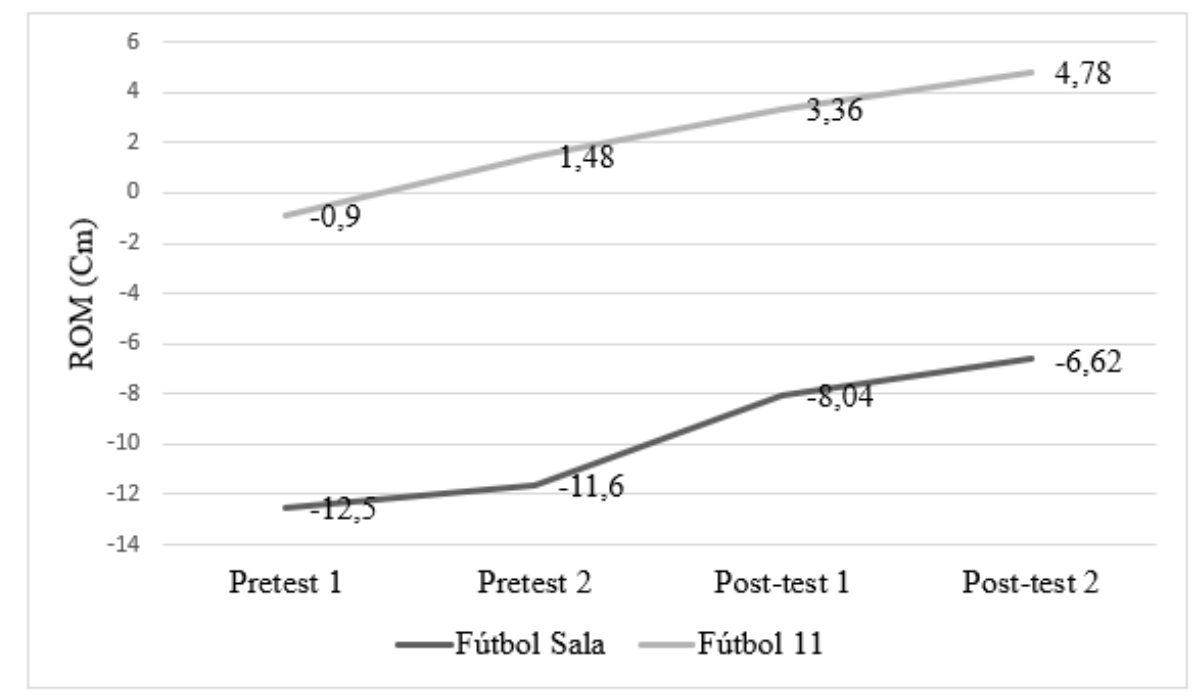

Figura 2. Comparación de medias del GE por modalidad deportiva.

\section{Discusión}

Como se observó en los resultados, se produce un aumento de ROM a medida que se ejecutan las mediciones tanto en el GC como en el GE, siendo más destacadas dichas ganancias en los sujetos que ejecutaron la SMR. Estos hallazgos ocurren del mismo modo en otros estudios similares como realizados por Do et al. (2018) y Grieve et al. (2015). En el estudio llevado a cabo por Merino et al. (2011a), al igual que en el presente, se observó un incremento flexibilidad en la musculatura isquiosural empleando la técnica de la pelota de golf sobre la superficie plantar en triatletas.

Las ganancias del ROM obtenidas no habiendo realizado el tratamiento SMR, pueden ser consecuencia del estiramiento estático tras las ejecuciones del SRT, que genera una tolerancia mayor al estiramiento y a una reducción en la rigidez muscular y tendinosa, tal y como indica Behm, Blazevich, Kay y McHugh (2016).

En cuanto al aumento en el ROM tras la SMR, Beardsley y Skarabot (2015) mencionan dos mecanismos neurofisiológicos que pueden originan dichas ganancias. El primero mecanismo de ellos involucra al "Órgano Tendinoso de Golgi" (GTO), el autor sugiere que una presión en este podría estimularlo y provocar un retardo en la velocidad de disparo que realiza la unidad motora, y por lo tanto reducir la tensión muscular. El segundo proceso implica a los mecanorreceptores, mencionando que una presión en los mecanorrecepto- res albergados en las fascias durante la SMR estimularía el sistema nervioso, dando lugar a una reducción de la tensión muscular. Otros autores como Capote et al. (2017) hace referencia a que la SMR genera un incremento de temperatura en las fascias, produciendo un estado más fluido en ellas y como consecuencia da lugar una ruptura de adherencias, restaurando así la extensibilidad de los tejidos blandos.

Estos mecanismos mencionados con anterioridad por Beardsley y Skarabot (2015) y Capote et al. (2017), unido a las interconexiones musculares (Ferreira, 2015) y en concreto a la interconexión entre las fascias plantares y la musculatura isquiosural que confirma Bolívar, Munuera y Padillo (2013), pueden ser las causas por la que tras ejecutar la SMR y liberar tensiones en las fascias plantares, se generan reducciones de tensiones derivando en la ganancia de flexibilidad de dicha musculatura.

Los datos obtenidos mediante la d de Cohen mostraron unos tamaños del efecto pequeños según Cohen (1988), tanto en el GC como en el GE, siendo este valor inferior en el GC. Los estudios similares mencionados con anterioridad (Do et al., 2018; Grieve et al., 2015; Merino et al., 2011a) no se ha empleado la d de cohen, por lo tanto, no es posible realizar una comparativa.

En las diferencias entre modalidades deportivas, se puede apreciar una tendencia a aumentar el ROM tanto en la modalidad de FU como en la de FS. Estas tendencias en el ROM tras los datos obtenidos en el pretest 2, pueden ser debido al 
estiramiento estático ejercido por el desarrollo del propio test, basándose en los efectos mencionados con anterioridad por Behm et al. (2016). Además, se observó un mayor aumento en aquella modalidad que más altos niveles de ROM previo presentaban (FU), pero estos datos deben ser analizados con cautela, ya que no se encuentran estudios comparen los efectos en muestras con niveles de ROM distintos.

Del mismo modo, se produce una tendencia a ganar ROM tras la ejecución de la SMR en ambas modalidades, tal como se observa en los estudios similares mencionados previamente (Do et al., 2018; Grieve et al., 2015; Merino et al., 2011a), aunque se debe resaltar un mayor aumento en aquella modalidad que posee menos ROM en las pruebas iniciales (FS). Al igual que se ha comentado anteriormente, no se han encontrados estudios comparen el efecto de la SMR en muestras de distintos niveles de ROM, por lo que los datos deben ser interpretados con cautela.

Estas ganancias de ROM tras la SMR, puede ser un buen método para corregir en cierta medida las posibles disfunciones en los deportistas, dando lugar a minimizar los factores lesivos derivados de la falta de flexibilidad en la musculatura isquiosural (Raya-González y Estévez-Rodríguez, 2016; Medeiros et al., 2016).

\section{Limitaciones}

La baja muestra de sujetos es una de las limitaciones principales del estudio.
El instrumento de medición empleado tiene validez para medir lo que se desea, y con una precisión media-alta, pero existen otros instrumentos que pueden utilizarse y obtener datos de mayor precisión.

Aunque el protocolo que se aplicó con el GE durante la SMR fue exacto con todos los sujetos, la intensidad indicada durante la SMR puede haber sido percibida de manera diversa por los sujetos, dando lugar a una posible disparidad en los resultados obtenidos.

\section{Perspectivas futuras}

En futuros estudios se podría investigar sobre un protocolo idóneo para realizar la SMR en deportistas. También podría ser conveniente observar la duración del efecto tras una SMR con pelota de golf en la fascia plantar, con el fin de desarrollar aplicaciones prácticas precompetitivas.

\section{Conclusiones}

Esta investigación ha aportado datos que corroboran los efectos inmediatos para el aumento de flexibilidad de la musculatura isquiosural, empleando para ello la SMR con la técnica de pelota de golf sobre la fascia plantar en deportistas federados de FS y FU.

Además, se concluye que después de ser sometidos los sujetos a la SMR, no parecen tener efectos agudos distintos una modalidad deportiva de otra (FU y FS).

\section{Referencias}

1. Ayala, F., Sainz. P., Croix M., y Santonja. F. (2012). Fiabilidad y validez de las pruebas sit-and-reach: revisión sistemática. Revista Andaluza de Medicina del Deporte, 5(2), 53-62. Doi: 10.1016/S1888-7546(12)700102.

2. Barnes, C., Archer. D. T., Hogg, B., Bush. M., y Bradley. P. S. (2014). The evolution of physical and technical performance parameters in the English Premier League. International Journal of Sports Medicine, 35(13), 1095-1100. Doi: 10.1055/s-0034-1375695.

3. Beardsley, C., y Skarabot, J. (2015). Effects of self-myofascial release: A systematic review. Journal of Bodywork and Movement Therapies, 19(4), 747-758. Doi: 10.1016/j.jbmt.2015.08.007.

4. Behm, D. G., Blazevich, A. J., Kay. A. D., y McHugh, M. (2016). Acute effects of muscle stretching on physical performance. range of motion. and injury incidence in healthy active individuals: a systematic review. Applied Physiology. Nutrition. and Metabolism, 41(1), 1-11. Doi: 10.1139/ apnm-2015-0235.

5. Bolívar, Y. A., Munuera, P. V., y Padillo, J. P. (2013). Relationship between tightness of the posterior muscles of the lower limb and plantar fasciitis. Foot \& Ankle International, 34(1), 42-48. Doi: 10.1177/1071100712459173.

6. Bush, M., Barnes, C., Archer, D. T., Hogg, B., y Bradley, P. S. (2015). Evolution of match performance parameters for various playing positions in the English Premier League. Human Movement Science, 39, 1-11. Doi: 10.1016/j.humov.2014.10.003.

7. Campignion, P. (2001). Cadenas musculares y articulares concepto G.D.S.
Aspectos biomecánicos. Alicante: Lenciana-Verdú Editores Independientes

8. Capote, G., Morales, R., Anthony, P., Analuiza, A., Fabián, E., González, G., ... y Raúl, A. (2017). Efectos de la autoliberación miofascial. Revisión sistemática. Revista Cubana de Investigaciones Biomédicas, 36(2), 271-283.

9. Cohen, J. (1988). Statistical Power Analysis for the Behavioral Sciences. Second Edition. Hillsdale, NJ: LEA.

10. DeBruyne, D. M., Dewhurst, M. M., Fischer, K. M., Wojtanowski, M. S., y Durall, C. (2017). Self-Mobilization Using a Foam Roller Versus a Roller Massager: Which Is More Effective for Increasing Hamstrings Flexibility? Journal of Sport Rehabilitation, 26(1), 94-100. Doi: 10.1123/ jsr.2015-0035.

11. Do, K., Kim, J., y Yim, J. (2018). Acute effect of self-myofascial release using a foam roller on the plantar fascia on hamstring and lumbar spine superficial back line flexibility. Physical Therapy Rehabilitation Science, 7(1), 35-40. Doi: 10.14474/ptrs.2018.7.1.35.

12. Ekstrand, J., Hägglund, M., y Waldén, M. (2011). Epidemiology of muscle injuries in professional football (soccer). The American Journal of Sports Medicine, 39(6), 1226-1232. Doi: 10.1177/0363546510395879.

13. Ekstrand, J., Waldén, M., y Hägglund, M. (2016). Hamstring injuries have increased by $4 \%$ annually in men's professional football. since 2001: a 13-year longitudinal analysis of the UEFA Elite Club injury study. British Journal of Sports Medicine, 50(12), 731-737. Doi: 10.1136/ bjsports-2015-095359. 
14. Ferreira, L. (2015). Influencia de la autoliberación miofascial versus estiramientos estáticos en un programa de entrenamiento de fuerza en miembros inferiores (tesis doctoral). Universitat de Valéncia, España.

15. Grieve, R., Goodwin, F., Alfaki, M., Bourton, A. J., Jeffries, C., y Scott, H. (2015). The immediate effect of bilateral self-myofascial release on the plantar surface of the feet on hamstring and lumbar spine flexibility: A pilot randomised controlled trial. Journal of Bodywork and Movement Therapies, 19(3), 544-552. Doi: 10.1016/j.jbmt.2014.12.004.

16. Joshi, D. G., Balthillaya, G., y Prabhu, A. (2018). Effect of remote myofascial release on hamstring flexibility in asymptomatic individuals - A randomized clinical trial. Journal of Bodywork and Movement Therapies, 22(3), 832-837. Doi: 10.1016/J.JBMT.2018.01.008.

17. Junker, D. H., y Stöggl, T. L. (2015). The Foam Roll as a Tool to Improve Hamstring Flexibility. Journal of Strength Conditioning Research, 29(12), 3480-3485. Doi: 10.1519/JSC.0000000000001007.

18. Kalichman, L., y Ben-David, C. (2017). Effects of self-myofascial release on myofascial pain. muscle flexibility. and strength: A narrative review. Journal of Bodywork and Movement Therapies, 21(2), 446-451. Doi: 10.1016/j.jbmt.2016.11.006.

19. MacDonald, G. Z., Button, D. C., Drinkwater, E. J., y Behm, D. G. (2014). Foam rolling as a recovery tool after an intense bout of physical activity. Medicine and Science in Sports and Exercise, 46(1), 131-142. Doi: 10.1249/MSS.0b013e3182a123db.

20. Martínez-Riaza, L., Herrero-González, H., López-Alcorocho, J. M., Guillen-García, P., y Fernández-Jaén, T. F. (2017). Epidemiology of injuries in the Spanish national futsal male team: a five-season retrospective study. British Journal of Sports Medicine, 2(1), e000180. Doi: 10.1136/bmjsem-2016-000180.

21. Mason, J. S., Crowell, M., Dolbeer, J., Morris, J., Terry, A., Koppenhaver, S., y Goss, D. L. (2016). The effectiveness of dry needling and stretching on hamstring flexibility: a randomized controlled trial. International Journal of Sports Physical Therapy, 11(5), 672-683. Doi: 10.1249/01.MSS.0000487690.64422.85.

22. Medeiros, D. M., Cini, A., Sbruzzi, G., y Lima, C. S. (2016). Influence of static stretching on hamstring flexibility in healthy young adults: Systematic review and meta-analysis. Physiotherapy Theory and Practice, 32(6), 438-445. Doi: 10.1080/09593985.2016.1204401.
23. Merino, R., López, I., Torres, G., Fernández, E. (2011b). Conceptos sobre flexibilidad y términos afines. Una revisión sistemática. Trances, 3(1), 1-32.

24. Merino, R., Mayorga, D., Fernández, E., y García, R. (2011a). The effect of sole self-massage on the extensibility of the back muscle chain in triathletes. A pilot study. Journal of Sport and Health Research, 3(1), 17-26.

25. Moon, J. H., Jung, J.-H., Won, Y. S., y Cho, H.-Y. (2017). Immediate effects of Graston Technique on hamstring muscle extensibility and pain intensity in patients with nonspecific low back pain. Journal of Physical Therapy Science, 29(2), 224-227. Doi: 10.1589/JPTS.29.224.

26. Okamoto, T., Masuhara, M., y Ikuta, K. (2014). Acute effects of self-myofascial release using a foam roller on arterial function. Journal of Strength Conditioning Research, 28(1), 69-73. Doi: 10.1519/ JSC.0b013e31829480f5.

27. Raya-González, J., y Estévez-Rodríguez, J. L. (2016). Revisión: Factores de riesgo asociados a la aparición de lesiones en el fútbol. Fútbolpf: Revista de Preparación física en el Fútbol 1889-5050, 21, 8-18.

28. Sainz, P., Cejudo, A., Ayala, F., y Santonja, F. (2015) Perfil óptimo de flexibilidad del miembro inferior en jugadoras de fútbol sala. Revista Internacional de Medicina y Ciencias de la Actividad Física y el Deporte, 15(60), 647-662. Doi: 10.15366/rimcafd2015.60.003.

29. Van Doormaal, M. C. M., van der Horst, N., Backx, F. J. G., Smits, D. W., y Huisstede, B. M. A. (2017). No Relationship Between Hamstring Flexibility and Hamstring Injuries in Male Amateur Soccer Players: A Prospective Study. American Journal of Sports Medicine, 45(1), 121-126. Doi: $10.1177 / 0363546516664162$.

30. Van Dyk, N., Farooq, A., Bahr, R., y Witvrouw, E. (2018). Hamstring and ankle flexibility deficits are weak risk factors for hamstring injury in professional soccer players: A prospective cohort study of 438 players including 78 injuries. American Journal of Sports Medicine, 46(9), 2203 2210. Doi: 10.1177/0363546518773057.

31. Witvrouw, E., Danneels, L., Asselman, P., D’Have, T., y Cambier, D. (2003). Muscle flexibility as a risk factor for developing muscle injuries in male professional soccer players. A prospective study. The American Journal of Sports Medicine, 31(1), 41-46. Doi: 10.1177/03635465030310011801. 